\title{
Capillary Discharge XUV Radiation Source
}

M. Nevrkla

A device producing Z-pinching plasma as a source of XUV radiation is described. Here a ceramic capacitor bank pulse-charged up to 100 $\mathrm{kV}$ is discharged through a pre-ionized gas-filled ceramic tube $3.2 \mathrm{~mm}$ in diameter and $21 \mathrm{~cm}$ in length. The discharge current has amplitude of $20 \mathrm{kA}$ and a rise-time of $65 \mathrm{~ns}$. The apparatus will serve as experimental device for studying of capillary discharge plasma, for testing X-ray optics elements and for investigating the interaction of water-window radiation with biological samples. After optimization it will be able to produce $46.9 \mathrm{~nm}$ laser radiation with collision pumped $\mathrm{Ne}$-like argon ions active medium.

Keywords: Capillary discharge, Z-pinch, XUV, soft X-ray, Rogowski coil, pulsed power.

\section{Introduction}

In the region of XUV (eXtended Ultra Violet) radiation, i.e. in the region of $\sim(0.2-100) \mathrm{nm}$ there are two significant sub-regions. The first is the water-window region (2.3-4.4) $\mathrm{nm}$. Radiation in this region is highly absorbed in carbon, but not much absorbed in water, so it is useful for observing organic samples in their native environment. The second is the region around $13.5 \mathrm{~nm}$. At this wavelength there are $\mathrm{Mo} / \mathrm{Si}$ multilayer mirrors with high reflectivity at a normal incidence angle, and thus radiation in this wavelength region it is convenient for industrial applications as XUV lithography. A typical $\mathrm{X}$-ray source, the Roentgen tube, is based on bremsstrahlung of electrons and produces a broad, continuous spectrum with intensity peaks on K-lines. If we are interested in a narrow spectrum of XUV radiation, synchrotron accelerators or FELs are suitable sources. However due to their huge dimensions, build and operation costs these devices are unobtainable for most laboratories. Another approach for obtaining XUV radiation involves electron transitions in plasma ions. Using this way, radiation with a line spectrum is created. Some electron transitions have noticeably higher probability than other transitions, and when suitable conditions arise in the plasma, these transitions become dominant and a significant peak can appear in the spectral region of interest. In addition, when the plasma forms a uniform column with length exceeding diameter in two orders, the pumping power is sufficiently high to ensure a high enough population on a meta-stable energy level, Spontaneous Amplified Emission (ASE) can appear. ASE has the properties of a laser. The Active medium of these lasers is created by laser-produced plasma or by capillary-produced plasma. The first capillary produced plasma XUV laser was made by Rocca et al. at Colorado University in 1994 [1]. In 2005, Heinbuch et al., from Rocca's team, produced a tabletop version of the laser [2].

\section{Discharge apparatus}

\subsection{Z-pinching capillary discharge}

In order to obtain radiation in the XUV region, the electron transitions have to proceed on highly ionized states. These states appear in plasma with a electron temperature of $T_{\mathrm{e}} \sim 100 \mathrm{eV}$. Such hot plasma with sufficient electron density $N_{\mathrm{e}}>10^{17} \mathrm{~cm}^{-3}$ is created by radial compression of a plasma column by the Lorentz force $\boldsymbol{F}_{\mathrm{L}}$ of a magnetic field $\boldsymbol{B}$ of flowing current $\boldsymbol{I}$ - a Z-pinch. First, the current flows along the walls of the capillary. The flowing current creates a magnetic field, which affects the charged particles of the plasma by a radial force. This force compresses the particles like a snowplow till the magnetic pressure is equilibrated by the plasma pressure. Schematic of a Z-pinching capillary discharge is shown in Fig. 1.

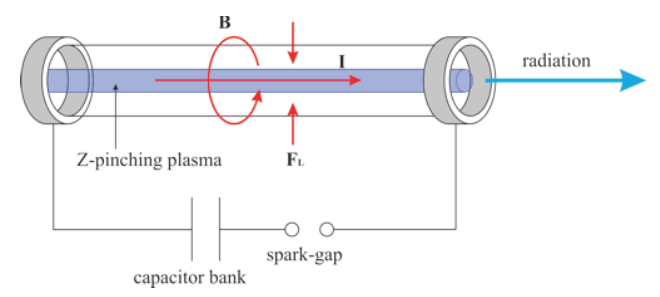

Fig. 1: Z-pinching capillary discharge

To obtain sufficient electron density and electron temperature, rapid compression is needed.

\subsection{Discharge circuit design}

Pulsed current of amplitude $\sim 10 \mathrm{kA}$ and an abrupt rise of $\mathrm{d} I / \mathrm{d} t>10^{11} \mathrm{As}^{-1}$ is needed for the rapid compression with a high compression rate, that is necessary for obtaining sufficiently dense and hot plasma. Our discharge circuit is realized by a ceramic capacitor bank discharged by closing of a spark-gap switch through ceramic capillary filled with gas. Because of the high $\mathrm{d} I / \mathrm{d} t$, all inductances in the circuit are undesirable because their impedance becomes dominant. Reduction of inductances in the circuit allows us to achieve high $\mathrm{d} I / \mathrm{d} t$ with a relatively small charging voltage. Fig. 2 shows the scheme of the apparatus.

A ceramic capacitor bank with a maximum capacity of $26.2 \mathrm{nF}$ is pulse charged by a 2-stage Marx generator and an RLC oscillating circuit up to $100 \mathrm{kV}$ (limited by the capacitors breakdown voltage). If the voltage on the capacitors exceeds the self-breakdown voltage of the spark gap, the gap is enclosed and the capacitors discharge through the capillary. This is the main discharge circuit. To reduce the inductance of this circuit, a close coaxial configuration was designed. The capillary is also shielded by a duralumin tube. This tube reduces the energy of magnetic the field around the capillary and thus reduces its inductance. Before the main discharge a 


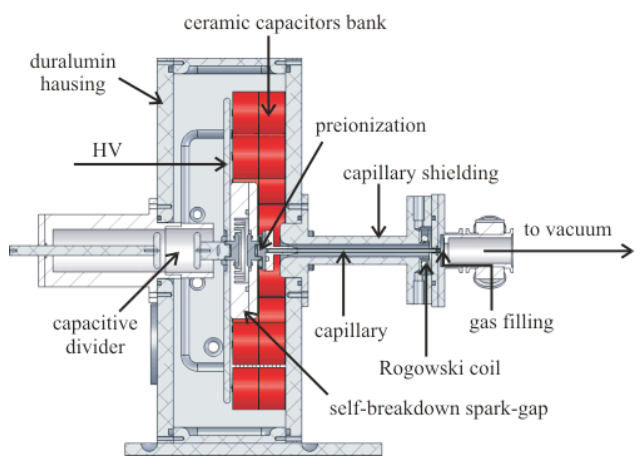

Fig. 2: Discharge apparatus

(30-40) A, (3-6) $\mu$ s long current pulse pre-ionizes the gas in the capillary and prepares a uniform conducting channel. The capillary is made of $\mathrm{Al}_{2} \mathrm{O}_{3}$, which is a material with low wall ablation. The ablation of the material from the capillary walls is an undesirable effect in the gas-filled capillary, which introduces impurities into the discharge plasma. The dimensions of capillary are: diameter $-3.2 \mathrm{~mm}$ and length $210 \mathrm{~mm}$. It is filled with gas through a hollow electrode on its grounded side. Radiation is also emitted through this hole. The XUV radiation is highly absorbed in any material, even a gas, so $3 \mathrm{~mm}$ from the capillary exit there is a $1.2 \mathrm{~mm}$ diameter aperture separating the discharge gas with working pressure (10-100) Pa from the high vacuum of 3 orders lower pressure. The charging voltage is measured by a $40 \mathrm{kV}$ Tektronix probe. Because the charging voltage of $100 \mathrm{kV}$ could damage the probe, a capacitive divider is used. This divider divides the voltage according to the ratio of the adjustable capacitor capacitance and the input capacitance of the probe. The current is measured using a Rogowski coil with RL integration. The breakdown voltage is adjusted by the nitrogen pressure in the spark-gap. The system is enclosed in a duralumin housing in order to reduce electromagnetic noise. Biodegraded oil is used to isolate the circuit and avoid unwanted breakdowns.

\subsection{Electrostatic analysis}

Electrostatic simulations in QuickField, finite elements analysis software, were performed in order to estimate spots predisposed to unwanted electrical break-downs. The input parameters were $100 \mathrm{kV}$ on the $\mathrm{HV}$ electrode, closed spark-gap, and constant resistivity along the capillary, i.e. a constant voltage drop between the electrodes of the capillary. The electric strength $E$ field picture in the region of $E=(0.02-2.00) 10^{7} \mathrm{~V} / \mathrm{m}$ with three problem spots is shown in Fig. 3.

During experiments without electro-isolating oil breakdowns really did occur between point $\mathrm{A}$ and the housing, and between point $\mathrm{B}$ and $\mathrm{C}$ in case of spark-gap switching into a non-preionized capillary. Formerly, when a DC charging voltage was used, these breakdowns occasionally also emerged in electro-isolating oil after exceeding $70 \mathrm{kV}$. After exceeding this voltage the main trigatron spark-gap also switched on unexpectedly. There was a need to change the DC charging design with a trigatron spark-gap to a pulse charging design with a self-breakdown spark-gap.

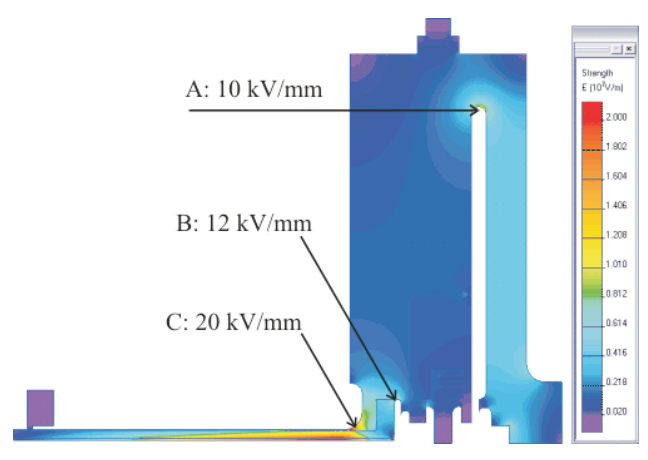

Fig. 3: QuickField electrostatic analysis

\subsection{Charging circuit and pre-ionization}

The ceramic capacitor bank is charged by a two-stage Marx generator and a RLC oscillating circuit. A simplified schematic of the circuit is shown in Fig. 4. The RLC circuit is formed by two charging capacitors with a capacity of $C_{C}=37.5 \mathrm{nF}$ in series, $\sim 1 \mathrm{mH}$ charging coil, charged capacitor bank $\mathrm{C}$, and by parasite resistivity. After closing the Marx generator, the voltage on the capacitor bank stabilizes approximately at a value of $U_{\mathrm{ST}}$, given by:

$$
U_{\mathrm{ST}}=U_{\mathrm{MARX}} \frac{C_{C}}{C+C_{C}},
$$

where $U_{\text {MARX }}$ is the voltage on charging capacitor $C_{C}$ after switching the Marx generator on. By charging through coil, the voltage on capacitor bank has a waveform of under-damped oscillations with a period of $\sim 25 \mu$ s (depending on the capacitor bank capacity), with the first maximum at $\sim 2 \times U_{\mathrm{ST}}$. In the case of $C \ll C_{C}$, the voltage on the capacitor bank can reach almost $160 \mathrm{kV}$, with a charging voltage of $40 \mathrm{kV}$ (doubled by Marx and again doubled in the coil).

The first stage of the Marx generator is used for pre-ionization of the capillary. The pre-ionization current is limited by the $1 \mathrm{k} \Omega$ resistor to $(30-40) \mathrm{A}$, and the duration of pre-ionization is given by the charging time of capacitor bank, i.e. by the time from Marx switching on to the self-breakdown spark-gap between the capacitors bank and capillary breakdown. This time is typically $(3-6) \mu$ s.

Formerly, the capacitor bank was charged by a DC power supply and discharged by the trigatron spark-gap switched by a commercial triggering unit. Pre-ionization circuit was separately charged by second power supply and switched by second commercial triggering unit. A breakdown of the capillary at the beginning of pre-ionization causes big electromagnetic noise. The triggering units were not resistant to this

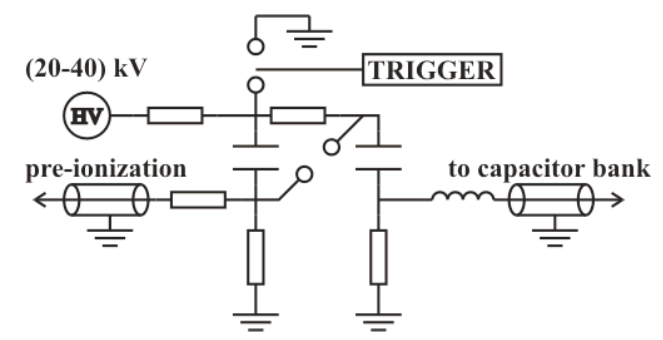

Fig. 4: Charging circuit 


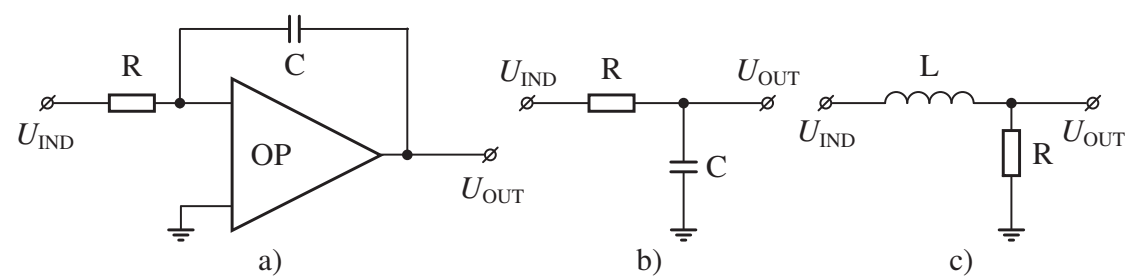

Fig. 5: Integrating circuits: a) active with OA, b) passive RC, c) passive RL

electromagnetic noise, and the main discharge was switched prematurely. This was another reason for turning to a pulse charging design with a self-breakdown spark-gap, one power supply, and one triggering unit.

\subsection{Rogowski coil}

The current in the capillary is measured by a Rogowski coil. This is a simple current probe based on the Faraday principle of electromagnetic induction. A toroidal coil with a returning loop is placed around the current conductor. In changing current, voltage $U_{\text {IND }}$ will be induced on the terminals of the coil, according to:

$$
U_{\mathrm{IND}}=-\frac{\mathrm{d} \Phi}{\mathrm{d} t}=-\mu_{0} S n \frac{\mathrm{d} I}{\mathrm{~d} t},
$$

where $\Phi$ is magnetic flux, $t$ is time, $\mu 0$ is permeability of vacuum, $S$ is cross-section of coil's loop, $I$ is measured current, and $n$ is number of loops of coil per unit length. As seen from (2), $U_{\text {IND }}$ is proportional to the change of current. In order to be proportional directly to measured current, $\mathrm{U}_{\text {IND }}$ has to be integrated. There are three basic types of integrating circuit, integration with an operating amplifier - Fig. 5.a, RC integration - Fig. 5.b, and RL integration - Fig. 5.c. Because of the big changes in the measured current, we do not need active integration with $\mathrm{OA}$. The measured current has a basic frequency around $3 \mathrm{MHz}$. A coil with RC integration should have a higher self-resonant frequency, in order to measure the current correctly. It is difficult to manufacture a coil, with sufficiently high $\mathrm{U}_{\mathrm{IND}}$ and sufficient high self-resonant frequency. The best solution for our case is RL integration.

The equivalent high-frequency circuit for a coil with RL integration is shown in Fig. 6. By solving this circuit and assuming $R_{\mathrm{D}} / L_{\mathrm{X}}<\omega<1 / R_{\mathrm{D}} C_{\mathrm{X}}$, where $R_{\mathrm{D}}$ is a small integrating resistor, $L_{\mathrm{X}}$ is coils inductance, and $C_{\mathrm{X}}$ is the inter-turns capacitance of the coil, and according to (2), and according to the coil inductance estimation:

$$
L_{\mathrm{X}}=\mu_{0} n V \frac{N}{l}=\mu_{0} n^{2} V
$$

where $\mu_{0}$ is permeability of air, $n$ is number of coil turns per unit length, $V$ is coil's inner volume, and $N$ is total number of coil turns, we can obtain the sensitivity of the coil:

$$
U_{\mathrm{OUT}}=\frac{\frac{R_{\mathrm{D}}}{R_{\mathrm{D}} \omega C_{\mathrm{X}}+1}}{\omega L_{\mathrm{X}}+\frac{R_{\mathrm{D}}}{R_{\mathrm{D}} \omega C_{\mathrm{X}}+1}} U_{\mathrm{IND}} \cong \frac{R_{\mathrm{D}}}{N} I .
$$

More on Rogowski coil theory is available in [3].

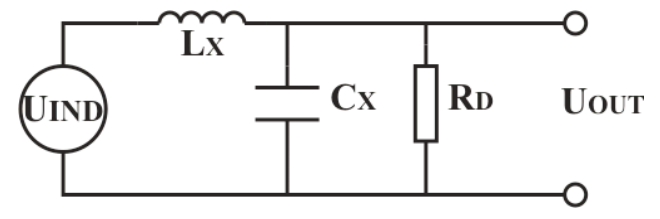

Fig. 6: High-frequency equivalent circuit of Rog. coil

Our Rogowski coil has the calculated properties:

low-frequency limit: $\quad f_{d}=\frac{1}{2 \pi} \frac{R_{\mathrm{D}}}{L_{\mathrm{X}}} \cong 29 \mathrm{kHz}$,

time resolution: $\quad t=\frac{l_{\mathrm{D}}}{c} \cong 10 \mathrm{~ns}$,

sensitivity:

$$
\frac{I}{U_{\mathrm{OUT}}}=\frac{N}{R_{\mathrm{D}}} \cong 137 \mathrm{AV}^{-1},
$$

where the time resolution is limited by the finite signal propagation along the coil wire length of $l_{\mathrm{D}}$. The coil was calibrated by placing it in the discharge system, where the capillary was replaced by a copper tube and the capacitor was discharged over a non-inductive $10 \Omega$ resistor. The discharge current was measured via differential voltage measurement on the resistor, and was compared with the voltage output of the coil. The experimentally determined sensitivity was:

$$
\frac{I}{U_{\text {OUT }}}=(130 \pm 8) \mathrm{AV}^{-1} \text {. }
$$

As seen, the theoretical sensitivity (7) is in good agreement with the measured sensitivity (8).

\section{Experimental results}

Here we present measurements of charging voltage, discharge current, output radiation, and pinch positions changing with respect to discharge gas pressure. Fig. 7 shows the charging voltage and the discharge current. The charging voltage is measured directly using a Tektronix $\mathrm{HV}$ probe and an HV probe behind the capacitor divider. The capillary is replaced by a copper tube. The capacitance of the capacitor bank is $11.2 \mathrm{nF}$.

Fig. 8 shows the measured current through an argon filled capillary at pressures $13 \mathrm{~Pa}, 40 \mathrm{~Pa}$, and $65 \mathrm{~Pa}$. The capacity of the bank is $15 \mathrm{nF}$ and the charging voltage is $75 \mathrm{kV}$. The pinch is observable as a drop on the current waveform. Fig. 8 shows, how the pinch moves forward in time with increasing pressure. The strongest pinch appears when the current reaches its maximum. For our experimental voltage and capacity it is at $40 \mathrm{~Pa}$. Fig. 8 also shows the XUV emission. The emission is measured by a vacuum diode with a gold cathode, $15 \mathrm{~cm}$ from 


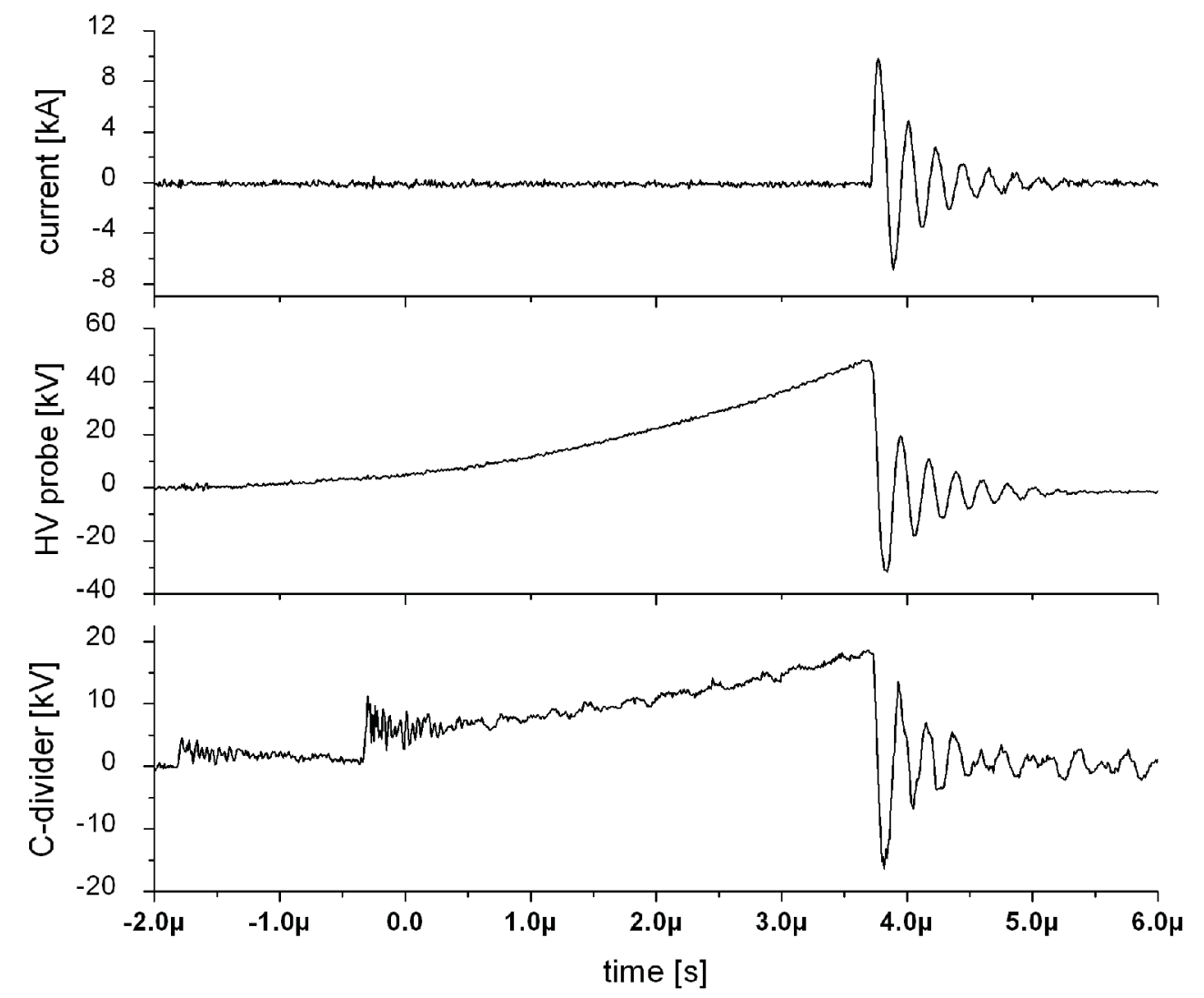

Fig. 7: Charging voltage and discharge current

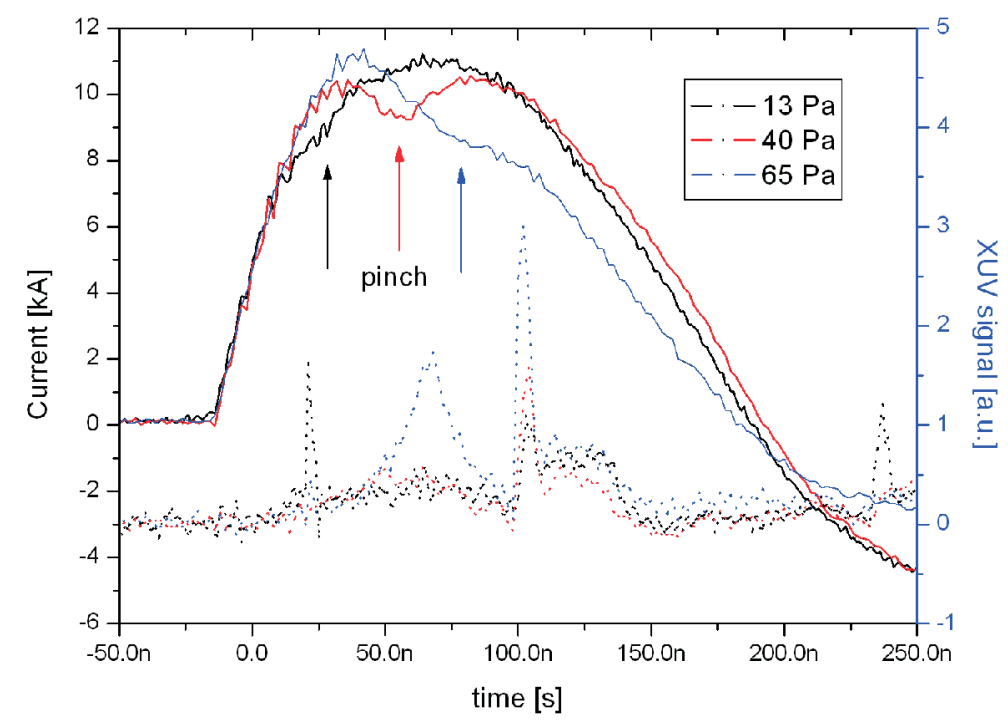

Fig. 8: Pinch and XUV radiation (current - solid line, XUV signal - dot-and-dash line)

the capillary end. Electrons, visible and UV radiation are filtered by a $0.8 \mu \mathrm{m}$ thick aluminum foil.

\section{Conclusion}

An apparatus for pinching capillary discharge production, as a source of XUV radiation, was designed and realized. The discharge current was measured using a calibrated Rogowski coil, the charging voltage was measured using a capacitive divider and a Tektronix HV probe, and the XUV output radiation was measured using a vacuum diode. The apparatus is in the final stage of construction. After completing the apparatus we would like to find the laser gain on Ne-like argon ions at a wavelength of $46.9 \mathrm{~nm}$. 


\section{Acknowledgment}

Research described in the paper was supervised by Dr. A. Jančárek. This research is supported by Ministry of Education CR grants 102/07/0275 and 202/08/H057, and internal grant of Czech Technical University in Prague CTU0910414. I also thank Dr. Vrba for consultations on the theoretical background of Z-pinching plasma and laser gain estimation.

\section{References}

[1] Rocca, J. J. et al.: Demonstration of a Discharge Pumped Table-Top Soft X-Ray Laser. Physical Review Letters, October 1994, Vol. 73 (1994), No. 16, p. 1236.

[2] Heinbuch, S., Grisham, M., Martz, D., Rocca, J. J.: Demonstration of a Desk-Top Size $46.9 \mathrm{~nm}$ Laser at
$12 \mathrm{~Hz}$ Repetition Rate. Optics Express, Vol. 13 (2005), No. 11, p. 4050-4055.

[3] Nevrkla, M.: Design and Realisation of Apparatus to Study Capillary Discharge in Argon: Master thesis. Prague: CTU, Faculty of Nuclear Sciences and Physical Engineering, 2008.

Michal Nevrkla

e-mail: michal.nevrkla@fjfi.cvut.cz

Department of Physical Electronics

Czech Technical University in Prague

Faculty of Nuclear Sciences and Physical Engineering Břehová 7

11519 Prague 1, Czech Republic 